\title{
Multilevel Modelling of Dispersed Multiphase Flows
}

\author{
J.A.M. Kuipers ${ }^{1}$ \\ 1 Twente University of Technology, Department of Chemical Engineering, PO Box 217, 7500 AE Enschede - The N etherlands \\ e-mail: J.A.M.Kuipers@ct.utwente.nl
}

\begin{abstract}
Résumé - Modélisation à plusieurs niveaux des écoulements polyphasiques dispersés - Les écoulements polyphasiques dispersés sont fréquemment rencontrés dans un grand nombre de procédés industriels importants dans les industries pétrolière, chimique, métallurgique et de l'énergie. L'extrapolation des équipements mettant en jeu des écoulements polyphasiques dispersés est très difficile à réaliser en raison de la complexité propre aux phénomènes d'écoulement (formation de bulles et d'amas dans les écoulements gaz/particule). Pendant les dix dernières années, des efforts importants de recherche ont été réalisés dans des laboratoires de recherche à la fois universitaires et industriels pour étudier les écoulements polyphasiques dispersés en utilisant des modèles basés sur des bilans à l'échelle microscopique (1 à 3). Malgré les limitations présentes, il est apparu clairement que cette approche offrait une puissante stratégie, complémentaire de l'expérimentation minutieuse, et l'on s'attend donc, dans un futur proche, à un développement important du rôle de la modélisation basée sur des modèles de bilans à l'échelle microscopique dans la conception et l'exploitation des réacteurs chimiques polyphasiques.
\end{abstract}

Dans cet article, le concept de modélisation à plusieurs niveaux est présenté et utilisé pour étudier les phénomènes d'écoulement à différentes échelles temporelles et spatiales. En outre, quelques résultats représentatifs sont présentés; ils mettent en relief les capacités de modélisation actuelles dans le domaine des écoulements à deux phases dispersées, gaz-liquide et gaz-solide. D'une façon générale, on distingue trois catégories de modèles différentes, à savoir des modèles adaptés à l'étude des écoulements polyphasiques à échelle microscopique, mésoscopique et macroscopique, chacun ayant ses avantages et ses inconvénients spécifiques. Il convient de souligner que le terme «particule» désigne des éléments dispersés tels que des particules solides, des gouttelettes ou des bulles.

Les modèles de Boltzmann sur réseau sont utiles pour étudier les phénomènes à une échelle fluideparticule nécessaire dans les modèles en milieu continu. En raison des limitations actuelles de la capacité des ordinateurs, l'approche par réseau de Boltzmann est limitée à l'étude du mouvement simultané de 1000 particules en suspension. Des modèles de particule discrets sont utiles pour étudier les phénomènes d'écoulement à une échelle mésoscopique et peuvent être utilisés pour développer des lois d'encadrement pour les interactions entre les particules et les interactions particule-paroi nécessaires aux modèles en milieu continu. Dans l'approche avec particules discrètes, l'on ne cherche pas à résoudre le champ d'écoulement au niveau de la sous-particule, et actuellement un nombre nettement plus important de particules peut être utilisé de ce fait dans le calcul (généralement 100 000). Il en résulte logiquement que, pour les lois de fermeture pour l'interaction fluide-particule, il doit être spécifié celles pouvant être obtenues à partir du modèle de Boltzmann sur réseau. Finalement, les connaissances obtenues sur la base du modèle de Boltzmann (fermeture pour l'interaction fluide-particule) et le modèle discret de particule (fermeture pour l'interaction entre les particules et l'interaction particule-paroi) peuvent être mises en application dans des modèles en milieu continu qui sont utiles pour étudier les phénomènes d'écoulement à l'échelle macroscopique.

Mots-clés : CFD (simulation numérique de la dynamique des fluides), lit fluidisé, colonne à bulles, eulérien, lagrangien. 


\begin{abstract}
Multilevel Modelling of Dispersed Multiphase Flows - Dispersed multiphase flows are frequently encountered in a variety of industrially important processes in the petroleum, chemical, metallurgical and energy industries. Scale-up of equipment involving dispersed multiphase flows is quite difficult which is mainly due to the inherent complexity of the prevailing flow phenomena (formation of bubbles and clusters in gas-particle flows). Especially during the last decade significant research efforts have been made in both academic and industrial research laboratories to study dispersed multiphase flows using detailed microbalance models (1 to 3). Despite current limitations it has become clear that this approach offers a powerful complementary strategy parallel to careful experimentation and it is therefore anticipated that the role of modelling based on detailed microbalance models for design and operation of multiphase chemical reactors will significantly expand in the near future.

In this paper the concept of multilevel modelling will be highlighted with which flow phenomena at different spatial and temporal scales can be studied. In addition some illustrative results will be presented which highlight current modelling capabilities in the area of dispersed gas-liquid and gassolid two-phase flows. Broadly speaking three different classes of models can be distinguished, each with its own specific advantages and disadvantages, namely models suited to study multiphase flows at the microscopic, mesoscopic and macroscopic level. It should be stressed here that "particle" corresponds to dispersed elements such as solid particles, droplets or bubbles.

Lattice Boltzmann models are useful to study flow phenomena at the microscopic level and can be used to develop closure laws for fluid-particle interaction needed in continuum models. Due to current limitations of computer capacity the lattice Boltzmann approach is limited to study the collective motion of 1000 suspended particles. Discrete particle models are useful to study flow phenomena at the mesoscopic level and can be used to develop closure laws for particle-particle and particle-wall interactions needed in continuum models. In the discrete particle approach one does not attempt to resolve the flow field at subparticle level and at present therefore far more (typically 100 000) particles can be allowed in the computation. As a logical consequence the closure laws for fluid-particle interaction have to be specified which can be obtained from the lattice Boltzmann model. Finally the knowledge obtained on the basis of the lattice Boltzmann model (closures for fluid-particle interaction) and the discrete particle model (closures for particle-particle and particle-wall interactions) can be implemented in continuum models which are useful to study flow phenomena at the macroscopic level.

Keywords: CFD (Computational Fluid Dynamics), fluidised bed, bubble column, Eulerian, Lagrangian.
\end{abstract}

\section{NOTATION}

$D$ lateral dimension, $\mathrm{m}$

$E(t)$ differential residence time distribution function, $\mathrm{s}^{-1}$

$e$ restitution coefficient

$F_{i} \quad$ force acting on $i$-th particle, $\mathrm{kg} \cdot \mathrm{m} / \mathrm{s}^{2}$

$\mathrm{g}$ gravitational force per unit mass, $\mathrm{m} / \mathrm{s}^{2}$

$L$ vertical dimension, $\mathrm{m}$

$m_{i}$ mass of $i$-th particle, $\mathrm{kg}$

$M_{k l}$ momentum source term due to interaction between phase $k$ and phase $l, \mathrm{~kg} /\left(\mathrm{m}^{2} \cdot \mathrm{s}^{2}\right)$

$p$ pressure, $\mathrm{Pa}$

$t$ time, $\mathrm{s}$

$u$ velocity, $\mathrm{m} / \mathrm{s}$

$U$ superficial gas velocity, $\mathrm{m} / \mathrm{s}$

$v_{i} \quad$ velocity of $i$-th particle, $\mathrm{m} / \mathrm{s}$

\section{G reek Symbols}

$\varepsilon \quad$ volume fraction

$\mu$ dynamic friction coefficient

$\rho$ density, $\mathrm{kg} / \mathrm{m}^{3}$ $\tau \quad$ stress tensor, $\mathrm{Pa}$

$\tau_{p}$ particle response time, $\mathrm{s}$

$\tau_{\mathrm{K}}$ Kolmogorov time scale, $\mathrm{s}$

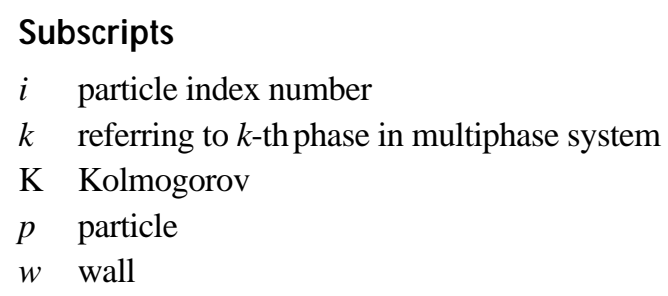

\section{Superscripts}

- vector quantity

\section{INTRO DUCTIO N}

In the last few decades Computational Fluid Dynamics (CFD) has become a very powerful and versatile tool for the analysis and solution of problems which are of considerable interest to the chemical engineer. Due to ongoing 
developments in both numerical algorithms for fluid flow and computer hardware, it can be anticipated that this tool will become increasingly important for the analysis and improvement of existing process equipment on one hand and design of new process equipment on the other hand. Traditionally the chemical engineering discipline has successfully developed and employed many semi-empirical strategies to solve problems of practical interest.

Prior to expanding on the theoretical framework for multiphase flow, the nature of these strategies will be highlighted using an example with relevance to fluid flow in chemical reactors.

\section{TRADITIO N AL APPRO ACHES FO LOW ED WITHIN CHEMICAL REACTION EN GIN EERIN G}

In the past, many powerful tools with a strong empirical base have been devised by chemical engineers to (approximately) solve extremely complicated problems encountered in chemical reaction engineering such as the design of process equipment (including chemical reactors) involving nonideal flow (Fig. 1). To facilitate design of chemical reactors in practice very often idealisation of the nonideal flow pattern has been applied using the concepts of plug flow and completely mixed flow. Very often the two idealised flow patterns, i.e. plug flow and completely mixed flow, do not occur in reality but nevertheless these concepts have proven valuable when dealing with the design of chemical reactors because the conversion obtained in these two extreme cases provides the boundaries for the conversion in a chemical reactor in which nonideal flow prevails. A well-known traditional approach adopted in the chemical engineering community is the characterisation of nonideal flow patterns by means of Residence Time Distribution (RTD) experiments where typically the response of a piece of process equipment is measured due to a disturbance of the inlet concentration of a tracer. From the measured response of the
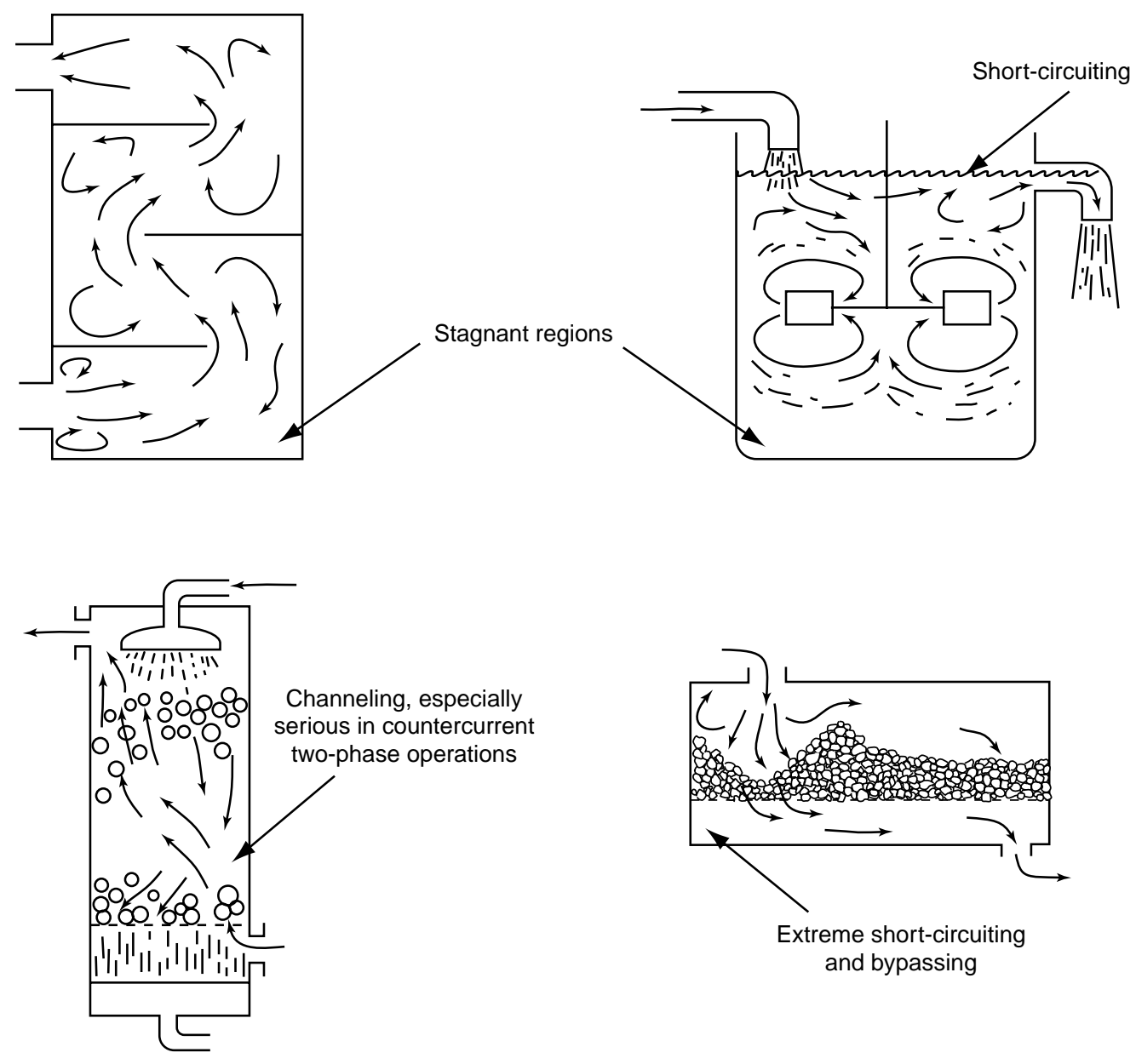

Figure 1

Some typical examples of nonideal flow patterns which can occur in process equipment (Levenspiel, 1962). 
system the differential RTD function $E(t)$ can be obtained, where $E(t) \mathrm{d} t$ represents the fraction of the volume elements with a residence time between $t$ and $t+\mathrm{d} t$. This function can subsequently be used to quantify the effect of the macroscopic flow pattern (i.e. the distribution of residence times of fluid elements or parcels passing through the reactor) on the conversion. Due to the advent of CFD the aforementioned approach can still be followed but now the $E(t)$ function can in principle be computed from the computed velocity distribution. Alternatively the species conservation equations can be solved simultaneously with the fluid flow equations and thereby the extent of chemical conversion can also be obtained directly without invoking the concept of RTD functions. Subsequently the theoretical framework for multiphase flow systems will be briefly outlined.

\section{THEO RETICAL FRAMEW O RK}

For each continuous phase $k$ present in a multiphase system consisting of $N$ phases, in principle conservation equations for mass, momentum and thermal energy can be applied. If one or more of the $N$ phases consist of solid particles, the Newtonian conservation laws for linear and angular momentum should be used instead. The resulting formulation of a multiphase system will be termed the local instant formulation. Through the specification of the proper initial and boundary conditions and appropriate constitutive laws for the viscous stress tensor the hydrodynamics of a multiphase system can in principle be obtained from the solution of the governing equations. However, for most systems of practical interest the analysis of multiphase systems on the basis of the local instant formulation is intractable, even for existing and near-future supercomputers, and consequently some kind of simplification must be made. From a computational point of view, this state of the art bears some resemblance with the problems encountered in Direct Numerical Simulation (DNS) of turbulent single-phase flows.

\subsection{Eulerian Approach}

The aforementioned simplification can be achieved through a continuum mathematical description of the multiphase system. The derivation of the continuum equations is usually based on spatial averaging techniques where the pointhydrodynamic variables are replaced by local averaged variables. For multiphase isothermal systems involving laminar flow the conservation equations for mass and momentum are respectively given by:

$$
\begin{gathered}
\frac{\partial}{\partial t}\left(\rho_{k}\right)+\left(\nabla \cdot \rho_{k} \bar{u}_{k}\right)=0 \\
\frac{\partial}{\partial t}\left(\rho_{k} \bar{u}_{k}\right)+\left(\nabla \cdot \rho_{k} \bar{u}_{k} \bar{u}_{k}\right)= \\
-\varepsilon_{k} \nabla p-\left(\nabla \cdot \varepsilon_{k} \tau_{k}\right) \\
+\sum_{l=1}^{l=N} \bar{M}_{k l}+\rho_{k} \overline{\mathrm{g}}
\end{gathered}
$$

where $\rho_{k}, u_{k}, \varepsilon_{k}$ and $\tau_{k}$ represent respectively the macroscopic density, velocity, volume fraction and viscous stress tensor of the $k$-th phase, $p$ the pressure, $M_{k l}$ the interphase momentum exchange rate between phase $k$ and phase $l$.

\subsection{Eulerian-Lagrangian Approach}

In multiphase systems involving one or more dispersed phases an alternative to the aforementioned complete continuum representation is possible by adopting a Lagrangian description for these phases. The advantages of this mixed Eulerian-Lagrangian approach are its greater generality and flexibility with respect to the incorporation of microscopic transport phenomena whereas its relatively high (compared to completely Eulerian approaches) computational load constitutes its most important disadvantage.

However, also from a computational point of view a mixed Eulerian-Lagrangian approach can offer certain advantages. If a Lagrangian description is adopted to represent the dispersed phase, for each individual particle (or bubble or droplet) an equation of motion is solved:

$$
\frac{\mathrm{d}}{\mathrm{d} t}\left(m_{i} \bar{v}_{i}\right)=\sum \bar{F}_{i}
$$

where $m_{i}, v_{i}$ represent respectively the mass and velocity of the $i$-th particle and $\Sigma F_{i}$ the sum of the forces acting on the $i$ th particle. Forces due to gravity, drag, virtual mass, vorticity in the continuous phases and electrical forces can be included in this term. The particle position vector is calculated from:

$$
\frac{\mathrm{d}}{\mathrm{d} t} \bar{x}_{i}=\bar{v}_{i}
$$

The solution of differential Equations (3) and (4) can be obtained with standard numerical integration techniques.

Depending on the volume fraction of the dispersed phase one-way coupling or two-way coupling between the dispersed phase and the continuous phase prevails. In systems involving (turbulent) multiphase flow at very small volume fraction of the dispersed phase, say smaller than $10^{-6}$, one-way coupling may be assumed. At such low volume fractions, the effect of the particles on the turbulence structure in the continuous phase is negligible while particleparticle interactions (i.e. collisions) do not play any role. For systems with higher volume fractions $\left(10^{-6}\right.$ to $\left.10^{-3}\right)$ the turbulence structure of the continuous phase is influenced by the dispersed phase while particle-particle interaction can still be neglected and two-way coupling between the phases has to be accounted for. With respect to the effect of the dispersed phase on the turbulence structure it can be mentioned that the ratio of the particle response time $\tau_{p}$ and the Kolmogorov time scale $\tau_{\mathrm{K}}$ determines whether the particles will enhance the production rate of turbulence energy $\left(\tau_{p} / \tau_{\mathrm{K}}>100\right)$ or increase the dissipation rate of turbulence energy $\left(\tau_{p} / \tau_{\mathrm{K}}<100\right)$. For still higher volume fractions of the dispersed phase particle-particle interactions 


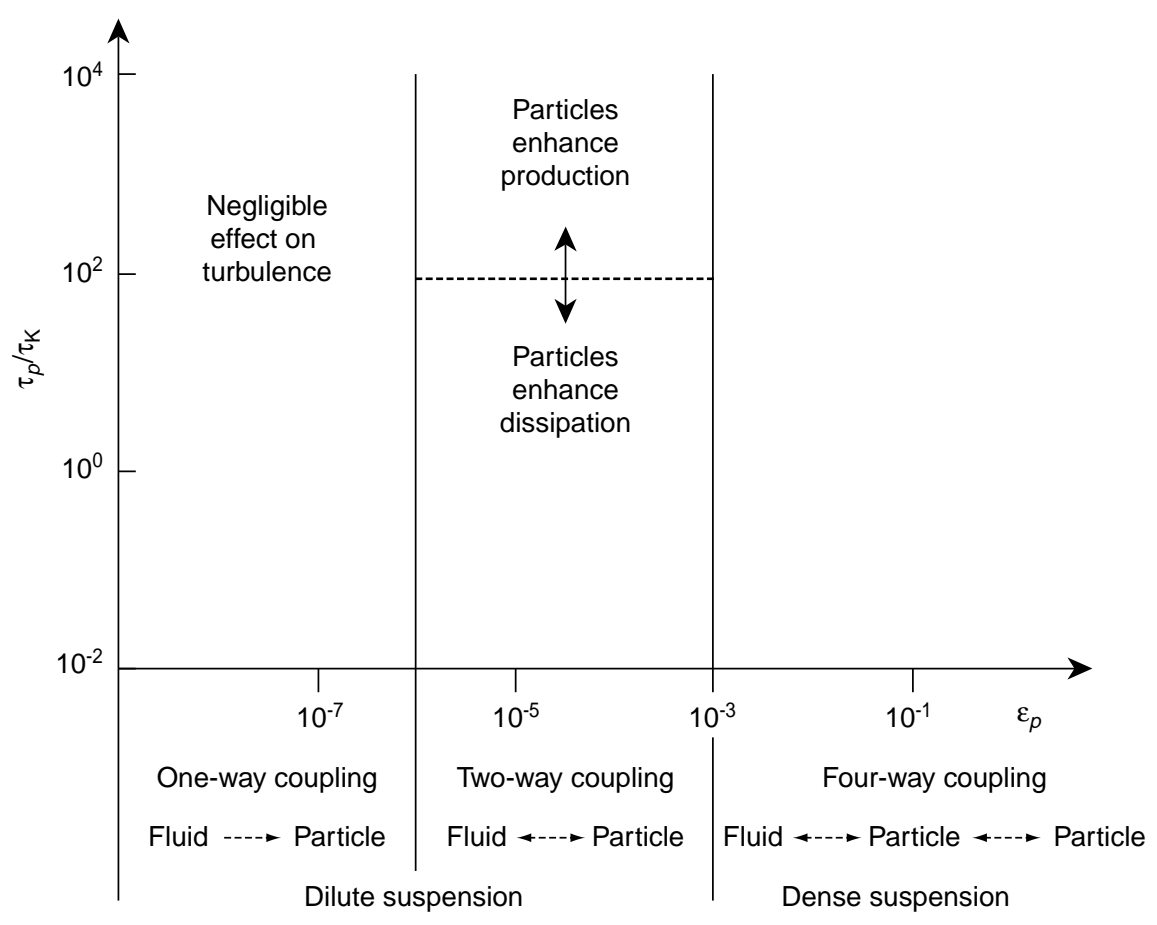

Figure 2

Map of flow regimes in particle-laden flows (adapted from Elgobashi, 1991).

(i.e. collisions) become important and four-way coupling has to be accounted for (Fig. 2). In this case an integrated modelling approach can be used combining features of Molecular Dynamics (MD), to effectively deal with the huge number of particle-particle and/or particle-wall collisions, and CFD to obtain the velocity distribution in the continuous phase (Section 4).

\section{CO MPUTATIO N AL STRATEG IES FO R THE EULERIAN-LAGRANGIAN APPROACH}

For dispersed multiphase flow, roughly speaking, three different situations and corresponding computational strategies can be distinguished (Fig. 3):

1 dilute flows where on the average less than one particle is present in a computational cell;

2 dense flows where a relatively high number of particles are present in a computational cell;

3 dilute or dense flows where a large number of computational cells are contained in a single particle,

where it should be mentioned that the dispersed phase could also consist of drops or bubbles which could, in principle, be deformable.

The situation for the first case arises when suspensions are relatively dilute and the particles are small. Depending on the

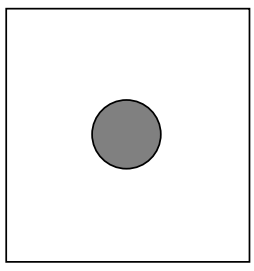

Case 1

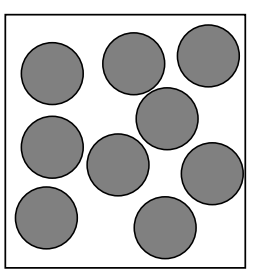

Case 2

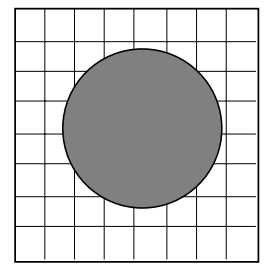

Case 3
Figure 3

Different cases which can be distinguished in modelling dispersed multiphase flow.

exact value of the volume fraction of the dispersed phase one-way coupling or two-way coupling prevails (Pan and Banerjee, 1996a). With one-way coupling particles are being moved in response to the fluid motion without feedback effects to the continuous phase, whereas in two-way coupling feedback effects are taken into account. As discussed in detail by Pan and Banerjee (1996a) care must be taken to correctly implement two-way coupling. Examples of oneway coupled calculations are those of Squires and Eaton (1990) and Pedinotti et al. (1992, 1993) who both used DNS to study respectively particle segregation in homogeneous isotropic turbulent flow and particle segregation in turbulent 
$t=0.0000 \mathrm{~s}$

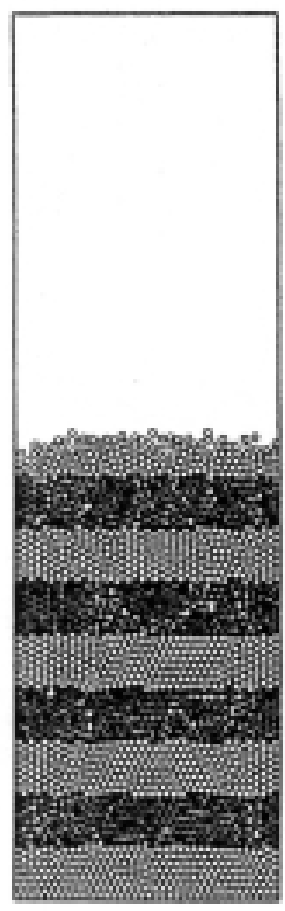

$t=0.0000 \mathrm{~s}$

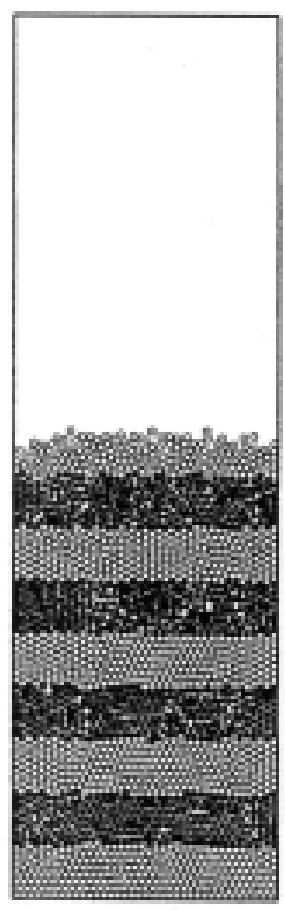

$t=0.1000 \mathrm{~s}$

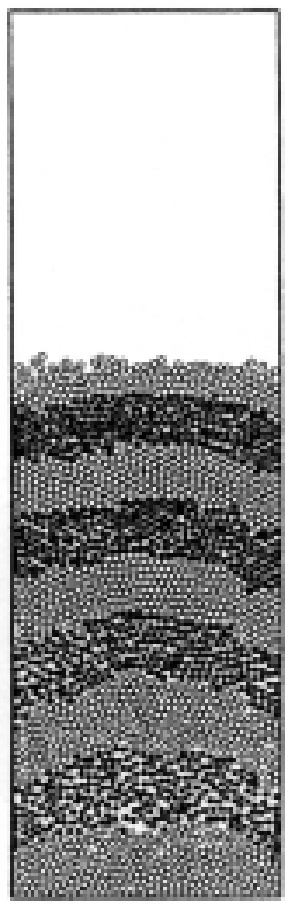

$t=0.1000 \mathrm{~s}$

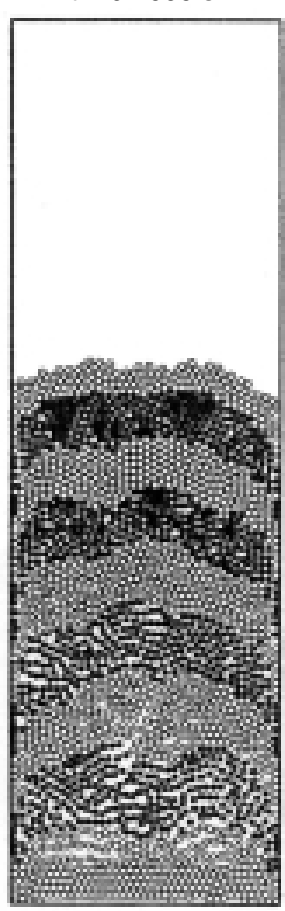

$t=0.2000 \mathrm{~s}$

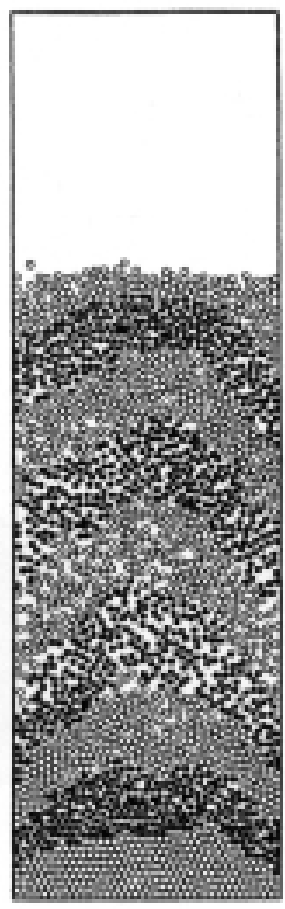

$t=0.2000 \mathrm{~s}$

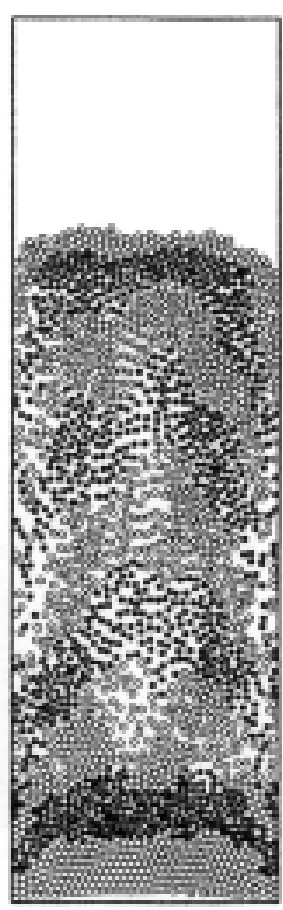

$t=0.3000 \mathrm{~s}$

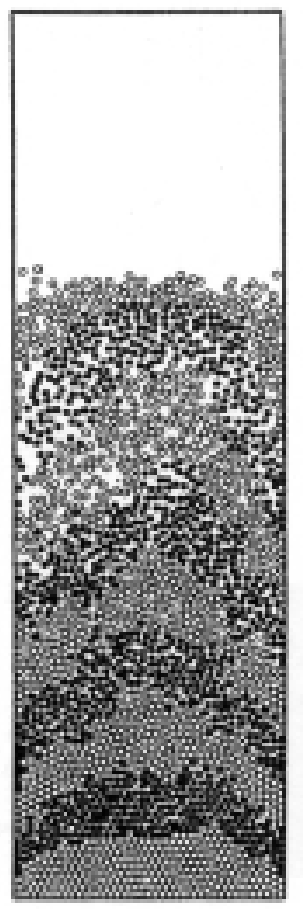

$t=0.3000 \mathrm{~s}$

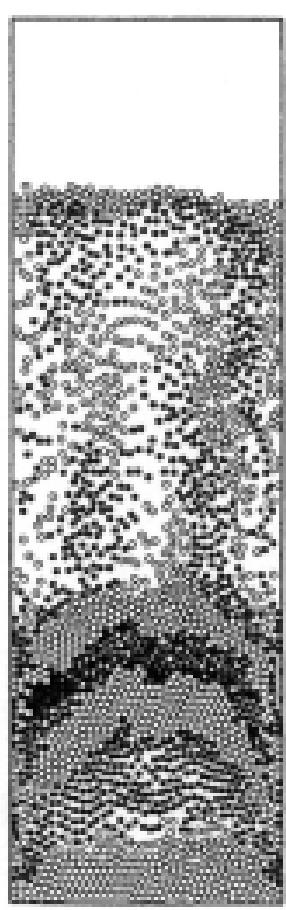

$t=0.4000 \mathrm{~s}$

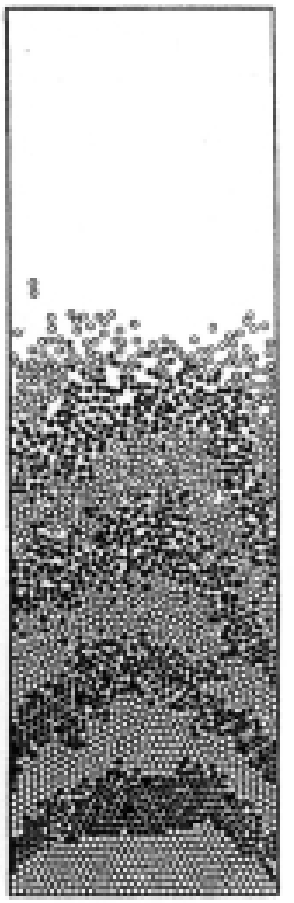

$t=0.4000 \mathrm{~s}$

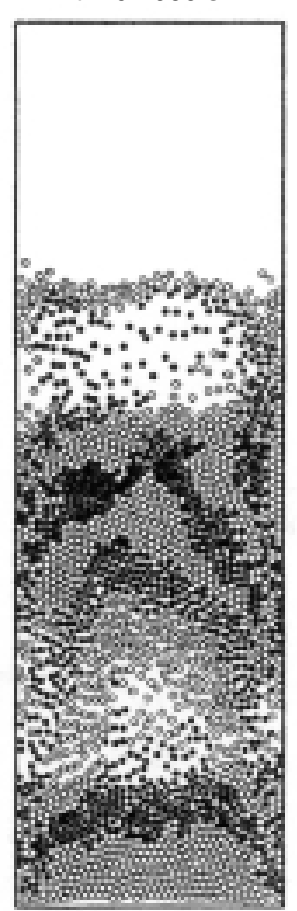

Figure 4

Snapshots of particle configurations for the simulation of slug formation with homogeneous inflow conditions in a two-dimensional gas-fluidised bed. Bottom: non-ideal particles $\left(e=e_{w}=0.9\right.$ and $\left.\mu=\mu_{w}=0.3\right)$, top: ideal particles $\left(e=e_{w}=1.0\right.$ and $\left.\mu=\mu_{w}=0.0\right)$. 
channel flow. For two-way coupling, different computational strategies are used, depending on the fact whether there is only one particle in a computational cell or many.

In the second case we deal with dense flows and in our computational strategy four-way coupling has to be accounted for since there is not only mutual interaction between the suspended particles and the continuous phase but also particle-particle interactions (i.e. collisions). The examples presented in Section 4 belong to this class of multiphase flows.

In the third case the relative size of the particles (with respect to the computational cells) is large enough so that they contain many hundred or even thousand of computational cells. It should be noted that the geometry of the particles is not exactly represented by the computational mesh and special, approximate techniques (i.e. body force methods) have to be used to satisfy the appropriate boundary conditions for the continuous phase at the particle surface (Pan and Banerjee, 1996b). Despite this approximate method, the empirically known dependence of the drag coefficient versus Reynolds number for an isolated sphere could be correctly reproduced using the body force method. Although these computations are at present limited to a relatively low number of particles they clearly have their utility because they can provide detailed information on fluid-particle interaction phenomena (i.e. wake interactions) in turbulent flows.

\section{RESULTS}

In this section results will be presented for dispersed gassolid (Section 4.1) and dispersed gas-liquid (Section 4.2) twophase flows where respectively gas-fluidised beds and bubble columns correspond to the industrially relevant multiphase reactors. In both cases four-way coupling has been taken into account.

\subsection{Gas-Fluidised Beds}

Hoomans et al. (1996) developed a discrete particle model of gas-fluidised beds where the two-dimensional motion of individual, spherical particles was directly calculated from the forces acting on them, accounting for particle-particle and particle-wall interactions and interaction with the interstitial gas phase. Their collision model is based on the conservation laws for linear and angular momentum and requires, apart from geometrical parameters, a restitution coefficient and a friction coefficient. Techniques which are well known within the field of MD were used to process a sequence of collisions. In fact the model developed by Hoomans et al. (1996) can be seen as a combination of a so-called Granular Dynamics (GD) approach for the colliding particles and a CFD approach for the gas phase percolating through the particles. Discrete particle models offer certain distinct advantages over two-fluid models (Kuipers et al., 1992) since they require no specific assumptions concerning the solids rheology. Furthermore, the incorporation of a particle size distribution can be accommodated with relative ease.

Figure 4 shows a sequence of particle configurations obtained from a discrete particle simulation of a twodimensional gas-fluidised bed where homogeneous inflow conditions were set at the bottom of the bed. In both simulations the same value for superficial gas velocity was used $(U=3.0 \mathrm{~m} / \mathrm{s})$ which corresponds to roughly 1.75 time the minimum fluidisation velocity. The number of particles was 2400 whereas the diameter and the density of the particles amounted respectively $4 \mathrm{~mm}$ and $2700 \mathrm{~kg} / \mathrm{m}^{3}$. The particle configurations shown in the bottom part of Figure 4 represent the predicted bed behaviour in case realistic values were used for the restitution and friction coefficients $(e=0.9, \mu=0.3$ ) whereas the particle configurations shown in the top part refer to ideal particles $(e=1, \mu=0)$. From a comparison of the results obtained for these two cases, it can clearly be seen that the global system behaviour, i.e. the occurrence of bubbles and slugs and the associated mixing rate, differs significantly between the two cases. These results clearly indicate that dissipative processes on microscale have a decisive effect on the global system dynamics.

\subsection{Bubble Columns}

Delnoij et al. (1997) developed a detailed hydrodynamic model for dispersed gas-liquid two-phase flow based on a mixed Eulerian-Lagrangian approach. Their model describes

TABLE 1

Parameter values used in the numerical simulations of Delnoij et al. (1997)

\begin{tabular}{l|c}
\hline Column width $(\mathrm{m})$ & 0.1750 \\
\hline Column height $(\mathrm{m})$ & \\
$L / D=1.0$ & 0.1750 \\
$L / D=2.0$ & 0.3500 \\
$L / D=4.8$ & 0.8400 \\
$L / D=7.7$ & 1.3475 \\
$L / D=11.4$ & 1.9950 \\
\hline Superficial gas velocity $(\mathrm{m} / \mathrm{s})$ & 0.035 \\
\hline Physical properties $($ air-water $)$ & \\
Density liquid $\left(\mathrm{kg} / \mathrm{m}^{3}\right)$ & 1000 \\
Viscosity liquid $(\mathrm{kg} /(\mathrm{m} \cdot \mathrm{s}))$ & 0.001 \\
Density gas $\left(\mathrm{kg} / \mathrm{m}^{3}\right)$ & 1.2 \\
Bubble diameter $(\mathrm{m})$ & 0.002 \\
\hline Number of computational cells in lateral direction & 20 \\
\hline Number of computational cells in vertical direction & \\
$L / D=1.0$ & 20 \\
$L / D=2.0$ & 40 \\
$L / D=4.8$ & 96 \\
$L / D=7.7$ & 154 \\
$L / D=11.4$ & 228 \\
\hline Time step (s) & 0.005 \\
\hline
\end{tabular}



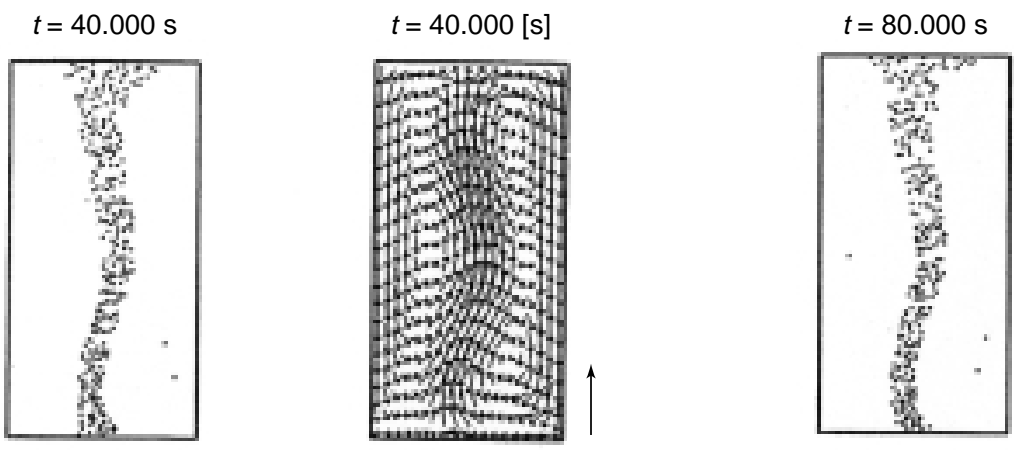

$t=80.000[\mathrm{~s}]$

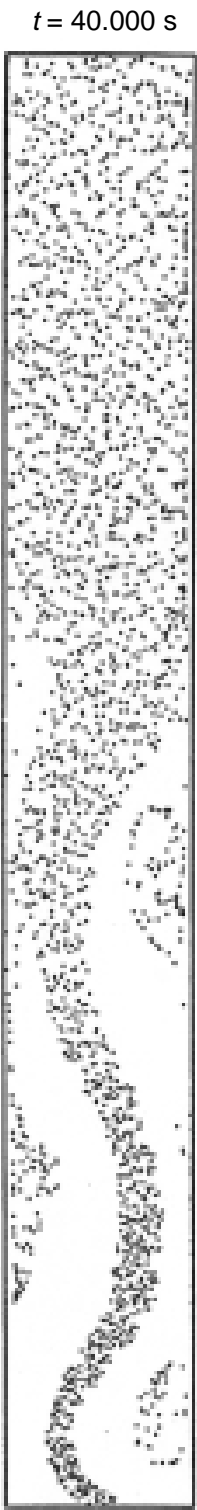

$t=40.000[\mathrm{~s}]$

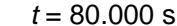

$t=80.000[\mathrm{~s}]$
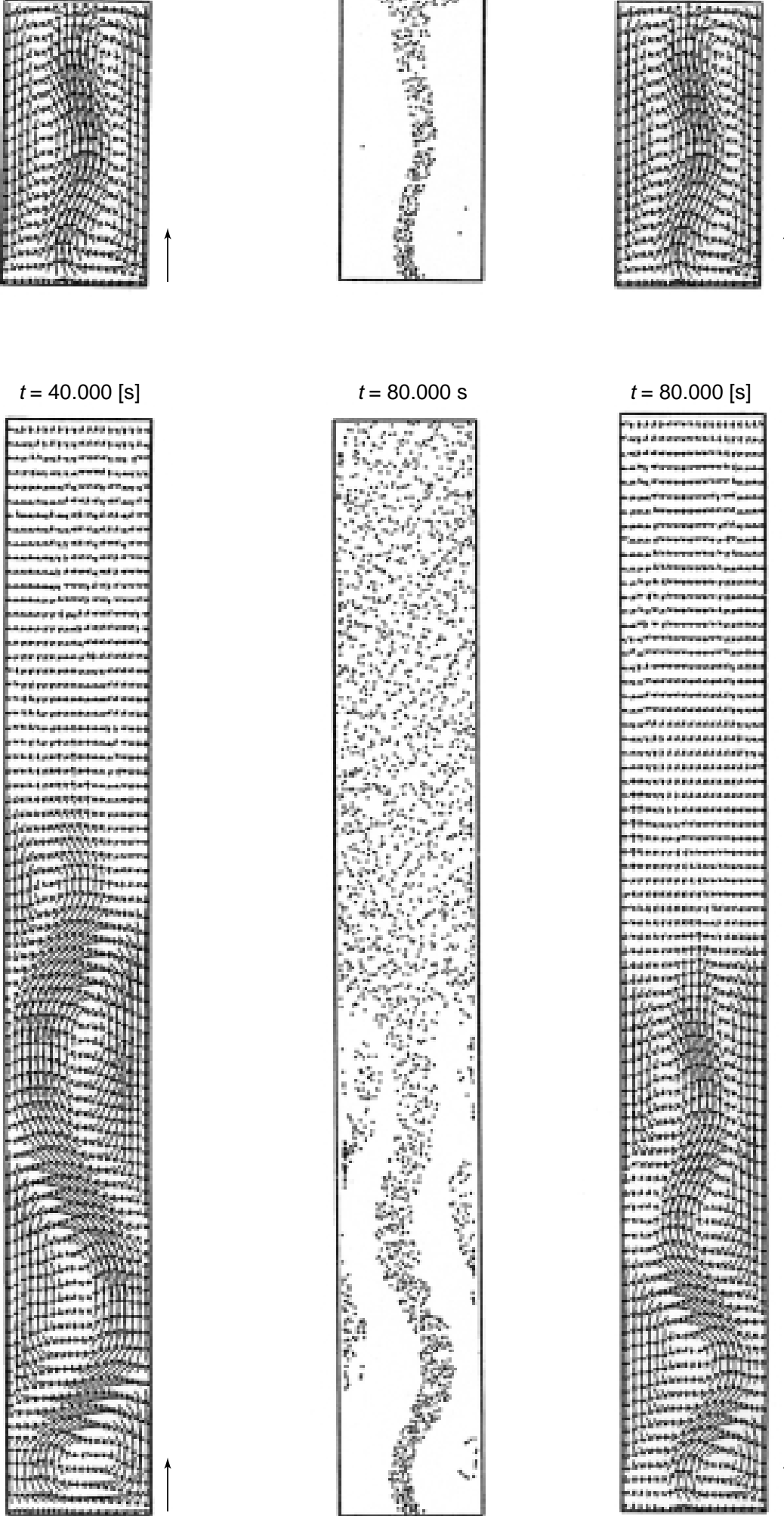

Figure 5

Effect of bubble column aspect ratio $L / D$ on the flow structure (top: $L / D=2.0$, bottom: $L / D=7.7$ ). The reference vector corresponds to a velocity of $1.0 \mathrm{~m} / \mathrm{s}$. 
the time-dependent motion of small, spherical gas bubbles in a bubble column operating in the homogeneous regime where all relevant forces acting on the bubble (drag, virtual mass, lift and gravity forces) were accounted for. Direct bubble-bubble interactions were accounted for via an interaction model which resembles the collision approach followed by Hoomans et al. (1996) to model gas-fluidised beds.

Delnoij et al. (1997) investigated the effect of the bubble column aspect ratio on the prevailing flow structure and compared their computational results with experimental data reported by Chen et al. (1989). The important parameters used in their numerical simulations are summarised in Table 1. Chen et al. (1989) reported a transition in the gasliquid flow pattern in case the aspect ratio of the column changed from 2 to 4 . For an aspect ratio of 2 the Gulf Stream type of liquid circulation was predicted whereas for an aspect ratio of 4 a highly dynamic liquid flow pattern with multiple vortices was computed. From computer animations Delnoij et al. (1997) could observe that these vortices were generated at the free surface. Furthermore these vortices were found to be positioned staggered with respect to each other in the column. In part these computational results were supported by Chen's findings. The only major difference with the experimental observations of Chen et al. (1989) was the fact that the aforementioned transition already occurred at an aspect ratio of 1 . This discrepancy is most likely due to the two-dimensional nature of the model developed by Delnoij et al. (1997). As an illustration in Figure 5 a few plots of instantaneous bubble configurations and liquid phase velocity fields are shown for bubble columns with an aspect ratio of 2.0 (top) and 7.7 (bottom). From this figure it can be clearly seen that the flow structure is significantly affected by the column aspect ratio.

\section{CONCLUSIONS AND FUTURE RESEARCH}

\section{Future Role of CFD in Chemical Reaction Engineering}

From the review presented in this paper it has hopefully become apparent that CFD offers great potential for the chemical engineer. It is expected that the role of CFD in the future design of chemical reactors will increase substantially and that it will assist to reduce the required experimental effort to develop industrial (multiphase) reactors.

\section{Directions for Future Research}

The variety and degree of complexity of systems encountered in industrial practice demand for an integrated modelling approach where models with increasing degree of sophistication should be used to feed models which invoke submodels with a strong empirical base. For dispersed multiphase flows, mixed Eulerian-Lagrangian models offer the possibility to develop closure laws, which can subsequently be used in a multifluid framework suited for the simulation of macroscopic systems of interest. In this connection also the lattice Boltzmann method and Stokesian dynamics should be mentioned, which offer great potential for simulation of concentrated suspensions in which hydrodynamic interaction has to be accounted for. Turbulence modelling of multiphase flow systems requires major attention in the near future. Also the development of closure laws for phenomena taking place in the vicinity of interfaces, such as coalescence, break-up and accumulation of impurities, should be considered in more detail. Once these requirements have been met, in principle it would be possible to predict among others flow regime transition and the spatial distribution of the phases with confidence, which is of utmost importance to the chemical engineer dealing with the design of (novel) multiphase reactors.

\section{REFERENCES}

Chen, J.J.J., Jamialahmadi, M. and Li, S.M. (1989) Effect of Liquid Depth on Circulation in Bubble Columns: A Visual Study. Chem. Eng. Res. Des., 67, 203-207.

Delnoij, E., Lammers, F.A., Kuipers, J.A.M. and van Swaaij, W.P.M. (1997) Dynamic Simulation of Dispersed Gas-Liquid Two-Phase Flow Using a Discrete Bubble Model. Chem. Eng. Sci., 52, 9, 1429-1438.

Elgobashi, S. (1991) Particle-Laden Turbulent Flows: Direct Numerical Simulation and Closure Models. Appl. Sci. Res., 48, 3-4, 301-304.

Hoomans, B.P.B., Kuipers, J.A.M., Briels, W.J. and van Swaaij, W.P.M. (1996) Discrete Particle Simulation of Bubble and Slug Formation in a Two-Dimensional Gas-Fluidised Bed: A Hard Sphere Approach. Chem. Eng. Sci., 51, 1, 99-110.

Kuipers, J.A.M., van Duin, K.J., van Beckum, F.P.H. and van Swaaij, W.P.M. (1992) A Numerical Model of Gas-Fluidized Beds. Chem. Eng. Sci., 47, 8, 1913-1924.

Levenspiel, O. (1962) Chemical Reaction Engineering, John Wiley \& Sons, New York.

Pan, Y. and Banerjee, S. (1996a) Numerical Simulation of Particle Interactions with Wall Turbulence. Phys. Fluids, 8, 10, 2733-2755.

Pan, Y. and Banerjee, S. (1996b) Numerical Investigation of the Effects of Large Particles on Wall-Turbulence. Phys. Fluids (submitted).

Pedinotti, S., Mariotti, G. and Banerjee, S. (1992) Direct Numerical Simulation of Particle Behavior in the Wall Region of Turbulent Flows in Horizontal Channels. Int. J. Mult. Flow, 18B, 927-941.

Pedinotti, S., Mariotti, G. and Banerjee, S. (1993) Effect of Reynolds Number on Particle Behavior Near Walls in Two-Phase Turbulent Flows. Proc. 5th. Int. Symp. Flow Mod. Turb. Meas., Presses Ponts et Chaussées, Paris, 425 sq.

Squires, K.D. and Eaton, J.K. (1990) Particle Response and Turbulence Modification in Isotropic Turbulence. Phys. Fluids, A2, 7, 1191-1203.

Final manuscript received in January 2000 